\title{
On the Relation between Mains-Side Current THD and DC Link Voltage Loop Gain in Grid-Connected Unity Power Factor Operating Converters
}

\author{
Alon Kuperman, Senior Member, IEEE
}

\begin{abstract}
It is well-known that attainable DC link voltage loop bandwidth in grid-connected converters operating with unity power factor is limited due to trade-off with $\mathrm{AC}$-side current total harmonic distortion (THD). The letter reveals that THD requirement directly imposes the value of voltage loop gain magnitude at double-grid frequency; therefore the dynamic performance may be improved without deteriorating the grid-side current quality by modifying the controller structure such that the loop gain magnitude at double-grid frequency and the crossover frequency are decoupled. Experimental results validate the revealed findings.
\end{abstract}

Index Terms-Grid-connected converters, THD, loop gain.

\section{INTRODUCTION}

In order to comply with strict regulations of grid power quality standards [1], AC/DC converters operating with nearunity power factor are utilized in mains-connected power conversion systems irrespective of power flow direction. As a result, instantaneous grid-side power contains both DC and double-grid-frequency pulsating components. On the other hand, DC-side power is pulsating component free in steadystate. Consequently, short-time energy storage element (typically realized by bulk DC link capacitors) is required in order to cope with instantaneous power misbalance [2]. Pulsating power component absorbed by the DC link capacitance generates voltage ripple proportional to system power rating and inversely correlated to the DC link capacitance value [3]. Grid-connected converters are typically regulated utilizing dual-loop control structure with DC link voltage and inductor current serving as outer and inner loop variables, respectively [4]. While the current loop bandwidth is typically high (limited by switching and sampling frequencies only), the voltage loop (utilizing PI or type-II regulator as DC link voltage controller [5]) usually attains a relatively low bandwidth (around $10 \mathrm{~Hz}$ [6], [7]) in order to limit the amount of DC link voltage ripple fed back into the loop since it deteriorates the grid-side current THD [8]. Quantitative design guidelines for voltage controller coefficients selection based on steady-state and transient performance merits were established in [9]. It was shown that limiting the amount of DC link voltage ripple fed back into the loop inevitable imposes crossover frequency reduction in case of PI controller utilization. This letter quantitatively reveals that grid-side current THD requirement actually imposes the value of the voltage loop gain magnitude at double-grid frequency. Hence, if an addition degree of freedom (i.e. a notch filter [10]) is introduced in the controller, it is possible to increase the crossover frequency without sacrificing the grid-side current THD.

\section{MAINS-CONNECTED POWER CONVERSION SYSTEM}

A typical mains-connected unity power factor operating AC/DC power conversion system is presented in Fig. 1(a), consisting of grid-interfacing converter (GIC), bulk DC link capacitance $C_{D C}$ and load-interfacing converter (LIC). Both converters are assumed ideal (in terms of efficiency) for simplicity (without loss of generality), utilizing independent control structures [11]. GIC employs a dual-loop control structure with outer (voltage) loop regulating the DC link voltage to a set point value $V_{D C}^{*}$ utilizing a controller $C_{V}(s)$ as voltage loop compensator, which calculates the desired gridside current magnitude $i_{G M}^{*}$. The latter is multiplied by unitygrid-voltage template $\sin \left(\omega_{G} t\right)$ to create grid-side current reference signal $i_{G}^{*}$, tracked by the inner (current) loop, as shown in Fig. 1(b) [11]. In practice, current loop bandwidth is significantly higher than the double-grid frequency, i.e. current loop may be accurately represented by complementary sensitivity function $T_{i}(s)=1$ up to $2 \omega_{G}$ [4].

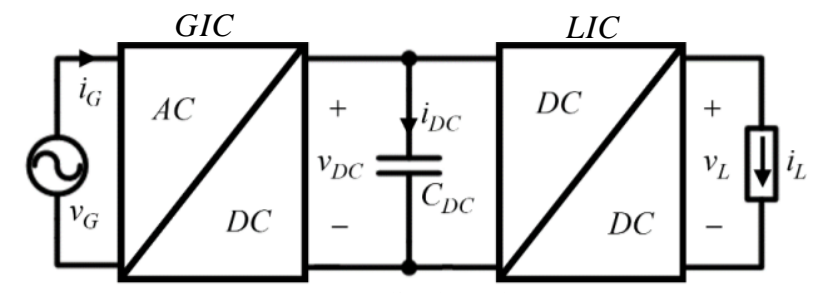

(a) Generalized topology.

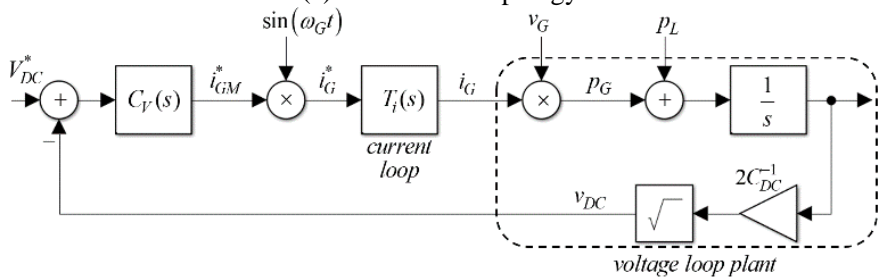

(b) Voltage loop structure.

Fig. 1. Typical dual-stage grid-connected AC/DC power conversion system.

In case the system operates correctly, steady-state grid-side voltage and current are described by

$$
\begin{aligned}
& v_{G}(t)=V_{G M} \sin \left(\omega_{G} t\right)+\underbrace{\sum_{n>1} V_{n} \sin \left(n \omega_{G} t\right)}_{\phi_{v}(t)}[\mathrm{V}] \\
& i_{G}(t)=V_{G M} \sin \left(\omega_{G} t\right)+\underbrace{\sum_{n>1} I_{n} \sin \left(n \omega_{G} t\right)}_{\phi_{i}(t)}[\mathrm{A}],
\end{aligned}
$$

with $\omega_{G}[\mathrm{rad} / \mathrm{s}]$ signifying grid frequency, $V_{G M}, I_{G M}$ representing grid voltage and current magnitudes, respectively, and $\phi_{v}(t), \phi_{i}(t)$ denoting residual harmonic content, inevitable present in practical systems so that [9] 


$$
\sqrt{\sum_{n>1} V_{n}^{2}}<<V_{G M}^{2}, \quad \sqrt{\sum_{n>1} I_{n}^{2}}<<I_{G M}^{2} .
$$

Instantaneous grid power is then given by

$$
\begin{aligned}
& p_{G}(t)=v_{G}(t) i_{G}(t)= \\
& \underbrace{0.5 V_{G M} I_{G M}}_{P_{G M}}-\underbrace{0.5 V_{G M} I_{G M}}_{P_{G M}} \cos \left(2 \omega_{G} t\right) \\
& +\underbrace{\phi_{v}(t) I_{G M} \sin \left(\omega_{G} t\right)+\phi_{i}(t) V_{G M} \sin \left(\omega_{G} t\right)+\phi_{v}(t) \phi_{i}(t)}_{\phi_{p}(t)} \\
& =P_{G M}+\underbrace{\left(-P_{G M}^{\left.\cos \left(2 \omega_{G} t\right)\right)}+\phi_{p}(t)[\mathrm{W}],\right.}_{\Delta p_{G}(t)}
\end{aligned}
$$

where $P_{G M}$ denotes average power, $\Delta p_{G}(t)$ represents lowfrequency content of pulsating (zero average) power component and $\phi_{p}(t)$ denotes residual harmonic content of pulsating power component. Taking into account (2), the root-meansquare value of $\phi_{p}(t)$ is much lower than that of $\Delta p_{G}(t)$ and the former is neglected hereinafter.

In steady state, load-side instantaneous power $p_{L}(t)$ is constant and energy balance holds, i.e.

$P_{G M}=P_{L}=p_{L}(t)=v_{L}(t) i_{L}(t) \Rightarrow I_{G M}=2 P_{L} V_{G M}^{-1}$

and

$p_{D C}(t)=v_{D C}(t) i_{D C}(t)=v_{D C}(t) C_{D C} \dot{v}_{D C}(t)=\Delta p_{G}(t)$.

Thus, steady-state DC link voltage is given by [3]

$$
v_{D C}(t) \approx V_{D C}^{*}-\Delta V_{D C} \sin \left(2 \omega_{G} t\right)
$$

with

$$
\Delta V_{D C}=\frac{P_{L}}{2 \omega_{G} V_{D C}^{*} C_{D C}}<<V_{D C}^{*} .
$$

Consequently, the desired grid-side current magnitude $i_{G M}^{*}$ is given in steady-state by (cf. (4), (6) and Fig. 1(b))

$$
\begin{aligned}
& i_{G M}^{*}(t)= \\
& \frac{2 P_{L}}{V_{G M}}+\frac{P_{L}}{2 \omega_{G} V_{D C}^{*} C_{D C}}\left|C_{V}\left(2 \omega_{G}\right)\right| \sin \left(2 \omega_{G} t+\arg C_{V}\left(2 \omega_{G}\right)\right)
\end{aligned}
$$

and corresponding grid-side current is obtained as

$$
i_{G}(t) \approx i_{G}^{*}(t)=i_{G M}^{*}(t) \sin \left(\omega_{G} t\right)=i_{G 1}(t)+i_{G 3}(t)
$$

with

$$
\begin{aligned}
& i_{G 1}(t)=2 P_{L} V_{G M}^{-1} \times \\
& \left(\sin \left(\omega_{G} t\right)+\frac{V_{G M}\left|C_{V}\left(2 \omega_{G}\right)\right|}{8 \omega_{G} V_{D C}^{*} C_{D C}} \cos \left(\omega_{G} t+\arg C_{V}\left(2 \omega_{G}\right)\right),\right. \\
& i_{G 3}(t)=-\frac{P_{L}\left|C_{V}\left(2 \omega_{G}\right)\right|}{4 \omega_{G} V_{D C}^{*} C_{D C}} \cos \left(3 \omega_{G} t+\arg C_{V}\left(2 \omega_{G}\right)\right) .
\end{aligned}
$$

Hence, grid-side current THD is given by the ratio of the third and the first harmonic root-mean-square values. As shown further on,

$$
\frac{V_{G M}\left|C_{V}\left(2 \omega_{G}\right)\right|}{8 \omega_{G} V_{D C}^{*} C_{D C}} \ll<1
$$

and hence grid-side current $T H D$ is given by

$$
T H D_{i} \cong \frac{V_{G M}}{8 \omega_{G} V_{D C}^{*} C_{D C}}\left|C_{V}\left(2 \omega_{G}\right)\right| .
$$

Typically, $T H D_{i} \ll<1$ is desired and hence (11) holds. Small- signal representation of Fig. 1(b) is shown in Fig. 2 [9] with linearized voltage loop plant dynamics given by

$$
\begin{aligned}
& \frac{d \tilde{v}_{D C}(t)}{d t}=\left(C_{D C} V_{D C}^{*}\right)^{-1} \times \\
& (\frac{V_{G M}}{2} \tilde{i}_{G M}(t)+\frac{P_{L}}{V_{G M}} \tilde{v}_{G M}(t)-\tilde{p}_{L}(t)-\underbrace{\tilde{p}_{G M}(t) \cos \left(2 \omega_{G} t\right)}_{\tilde{p}_{G M}(t)}),
\end{aligned}
$$

where $\tilde{x}$ denotes the small-signal term of variable $X$. According to Fig. 2, control-to-output transfer function of linearized voltage loop plant is given by

$$
P_{V}(s)=\left.\frac{\tilde{v}_{D C}(s)}{\tilde{i}_{G M}(s)}\right|_{\tilde{v}_{G M}=0, \tilde{p}_{L}=0}=\frac{V_{G M}}{2 C_{D C} V_{D C}^{*}} \frac{1}{s}
$$

and corresponding loop gain is obtained as

$$
L_{V}(s)=C_{V}(s) P_{V}(s)=C_{V}(s) \frac{V_{G M}}{2 C_{D C} V_{D C}^{*}} \frac{1}{s} .
$$

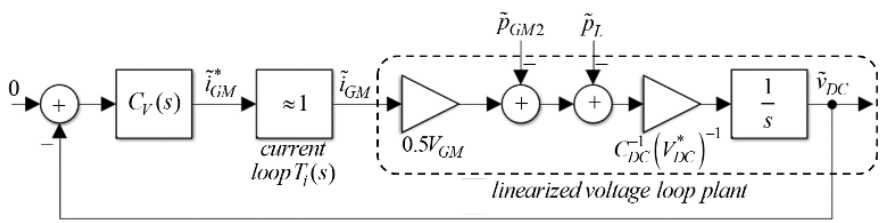

Fig. 2. Linearized voltage loop structure.

The value of voltage loop gain magnitude at $2 \omega_{G}$ is found as

$\left|L_{V}\left(2 \omega_{G}\right)\right|=\frac{V_{G M}}{4 \omega_{G} C_{D C} V_{D C}^{*}}\left|C_{V}\left(2 \omega_{G}\right)\right|$.

Comparing (16) with (12), there is

$\left|L_{V}\left(2 \omega_{G}\right)\right|=2 \cdot T H D_{i}$

irrespectively of voltage controller type. Hence, in order to keep the grid-side current THD below a desired value $T H D_{i}^{*}$, the controller $C_{V}(s)$ must be designed so that

$$
\left|L_{V}\left(2 \omega_{G}\right)\right| \leq 2 \cdot T H D_{i}^{*} \Rightarrow\left|C_{V}\left(2 \omega_{G}\right)\right| \leq \frac{8 \omega_{G} C_{D C} V_{D C}^{*}}{V_{G M}} T H D_{i}^{*} .
$$

On the other hand, load power (i.e. disturbance)-to-output transfer function is given by (cf. Fig. 2)

$$
D_{V}(s)=\left.\frac{\tilde{v}_{D C}(s)}{\tilde{p}_{L}(s)}\right|_{\tilde{v}_{G M}=0, \tilde{I}_{G M}=0}=\frac{2}{V_{G M}} \frac{P_{V}(s)}{1+C_{V}(s) P_{V}(s)} .
$$

It may then be concluded that the controller magnitude is bounded by $T H D_{i}^{*}$ only at $2 \omega_{G}$ (cf. (18)) and should be maximized elsewhere to improve disturbance rejection as long as the desired voltage loop phase margin value $P M_{V}^{*}$ is satisfied.

\section{VALIDATION}

In order to validate the revealed finding experimentally, a $500 \mathrm{~W}$ power factor correction rectifier (PFCR) shown in Fig. 3 was employed. The control system was implemented digitally using TMS320F28335 DSP-based control card. Average current control at a fixed switching frequency of $150 \mathrm{kHz}$ was adopted as the inner loop. The system was fed by APS-7100 Gwinstek programmable $\mathrm{AC}$ power source set to operate as 50 $\mathrm{Hz}, 230 \mathrm{~V}$ mains and terminated by M9715B Maynuo DC electronic load set to operate in constant power mode. Total DC link capacitance and reference voltage were set to $C_{D C}=385 \mu \mathrm{F}$ 
and $V_{D C}^{*}=400 \mathrm{~V}$, respectively. The desired performance merits pair was selected as

$$
P M_{V}^{*}=40^{\circ}, T H D_{i}^{*}=0.05 \text {, }
$$

i.e. $\left|L_{V}\left(2 \omega_{G}\right)\right|$ must remain lower or equal to 0.1 (or $-20 \mathrm{~dB}$ ). In addition, typical mains frequency uncertainty of $1 \%$ is assumed (i.e. $\omega_{G}$ may reside between $2 \pi \cdot 49.5 \mathrm{rad} / \mathrm{s}$ and $2 \pi \cdot 50.5 \mathrm{rad} / \mathrm{s}$ ).

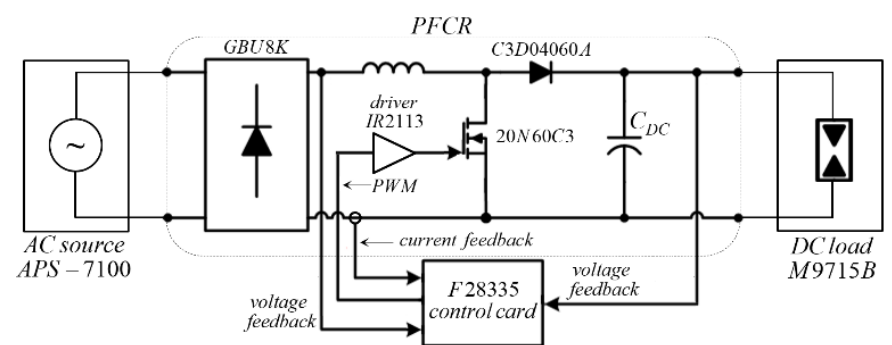

Fig. 3. Experimental setup.

A. PI controller

Typically, PI controller

$$
C_{V 1}(s)=K_{1} \frac{\tau_{1} s+1}{s}
$$

is adopted in industrial designs. Combining (15), (18), (20) and (21), coefficients of (21) are obtained as

$$
K_{1}=8.7, \quad \tau_{1}=0.007 \text {. }
$$

Bode diagram of corresponding voltage loop gain $L_{V I}(s)=$ $C_{V I}(s) P_{V}(s)$ is depicted in Fig. 4. It is well-evident that the values of loop gain magnitude at $99 \mathrm{~Hz}-101 \mathrm{~Hz}$ and phase margin comply with the desired performance merits pair. The crossover frequency of $\omega_{C l} \approx 2 \pi \cdot 17 \mathrm{rad} / \mathrm{s}$ is attained in this case.

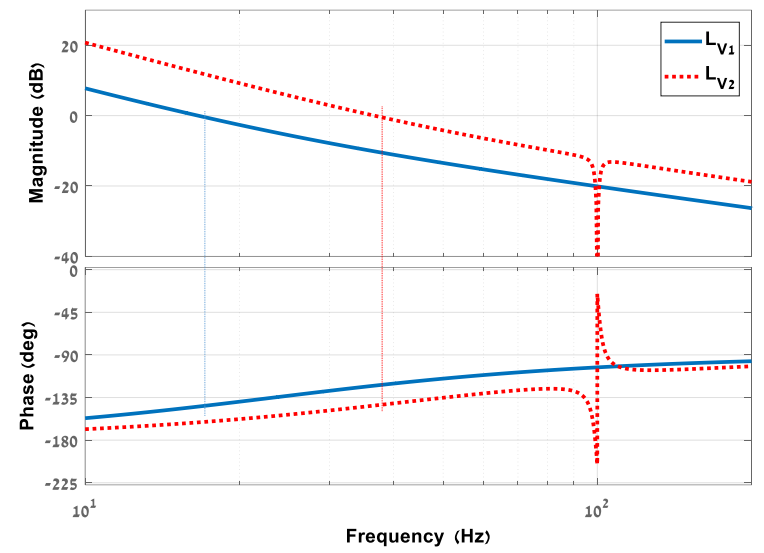

Fig. 4. Bode diagrams of voltage loop gain with PI and PI+Notch controllers.

\section{B. PI+Notch controller}

In order to increase the crossover frequency of voltage loop gain while complying with the desired performance merits pair, PI+Notch controller

$$
C_{V 2}(s)=K_{2} \frac{\tau_{2} s+1}{s} \frac{s^{2}+\left(2 \omega_{G}\right)^{2}}{s^{2}+4 \xi \omega_{G} s+\left(2 \omega_{G}\right)^{2}}
$$

may be adopted, providing an additional degree of freedom to pure PI controller (21). Combining (15), (18), (20) and (23), coefficients of (23) are obtained as

$$
\xi=0.019, \quad K_{2}=40, \quad \tau_{2}=0.0035 \text {. }
$$

Bode diagram of corresponding voltage loop gain $L_{V 2}(s)=$ $C_{V 2}(s) P_{V}(s)$ is depicted in Fig. 4, indicating that while the desired performance merits pair is obeyed, the crossover frequency $\omega_{C 2}=2 \pi \cdot 36 \mathrm{rad} / \mathrm{s} \approx 2 \cdot \omega_{C 1}$ is achieved. Since a twofold bandwidth increase is attained, similar improvement of DC link voltage deviation from reference value as a result of load power step is expected as well.

\section{Experimental results}

During experiments, steady-state grid-side current spectra was recorded and corresponding THD was measured. Moreover, system response to $0 \%$ - to $-100 \%$ load power step was logged to quantify the load rejection by means of DC link voltage deviation from set point value. The results are shown in Figs. 5 and 6 , respectively, for the worst case of $\omega_{G}=2 \pi \cdot 49.5$ $\mathrm{rad} / \mathrm{s}$. It is well-evident that PI+Notch controller utilization allowed to attain a two-fold load rejection improvement for the same value of $T H D_{i}$, as expected.

\section{CONCLUSION}

It was revealed in the letter that grid-side current THD requirement in grid-connected converters operating with unity power factor actually imposes the value of the voltage loop gain magnitude at double-grid frequency. Hence, it was shown that introducing an addition degree of freedom in the controller allows to decouple the loop gain magnitude at double-grid frequency and the crossover frequency thus increase the attainable DC link voltage loop crossover frequency without sacrificing the THD. Experimental results were given, accurately validating the revealed findings.
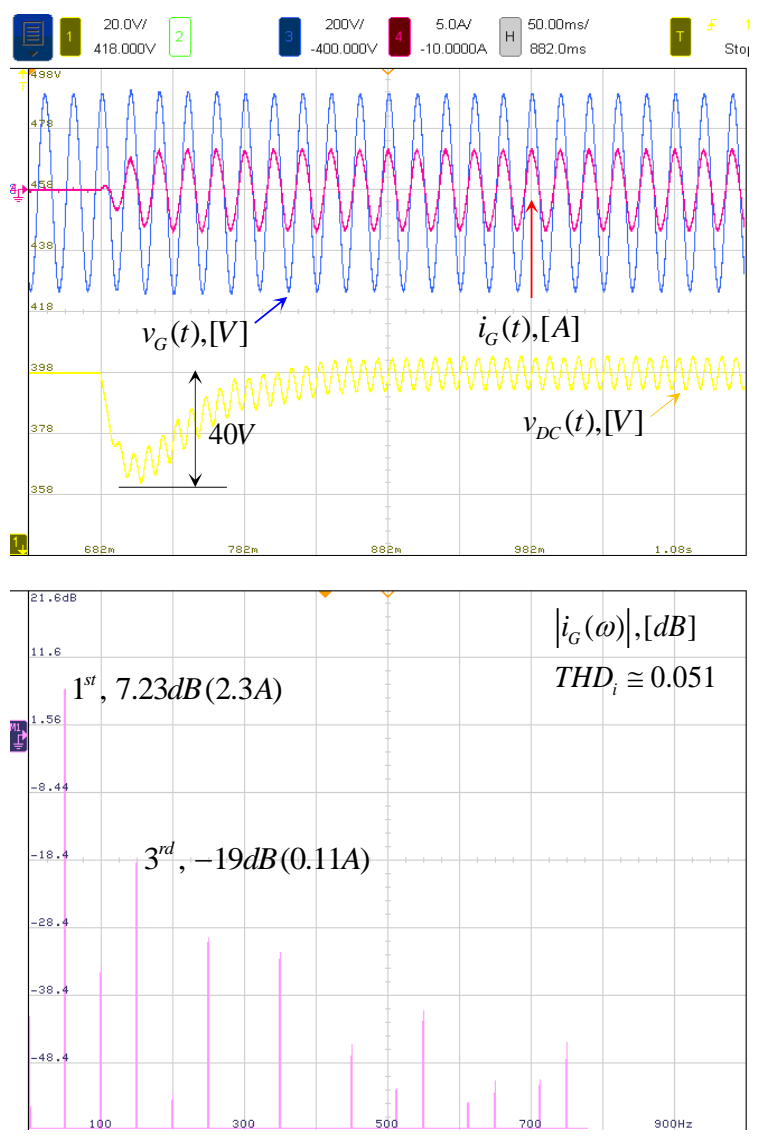

Fig. 5. System response to $0 \%$-to- $100 \%$ load step with PI controller. 

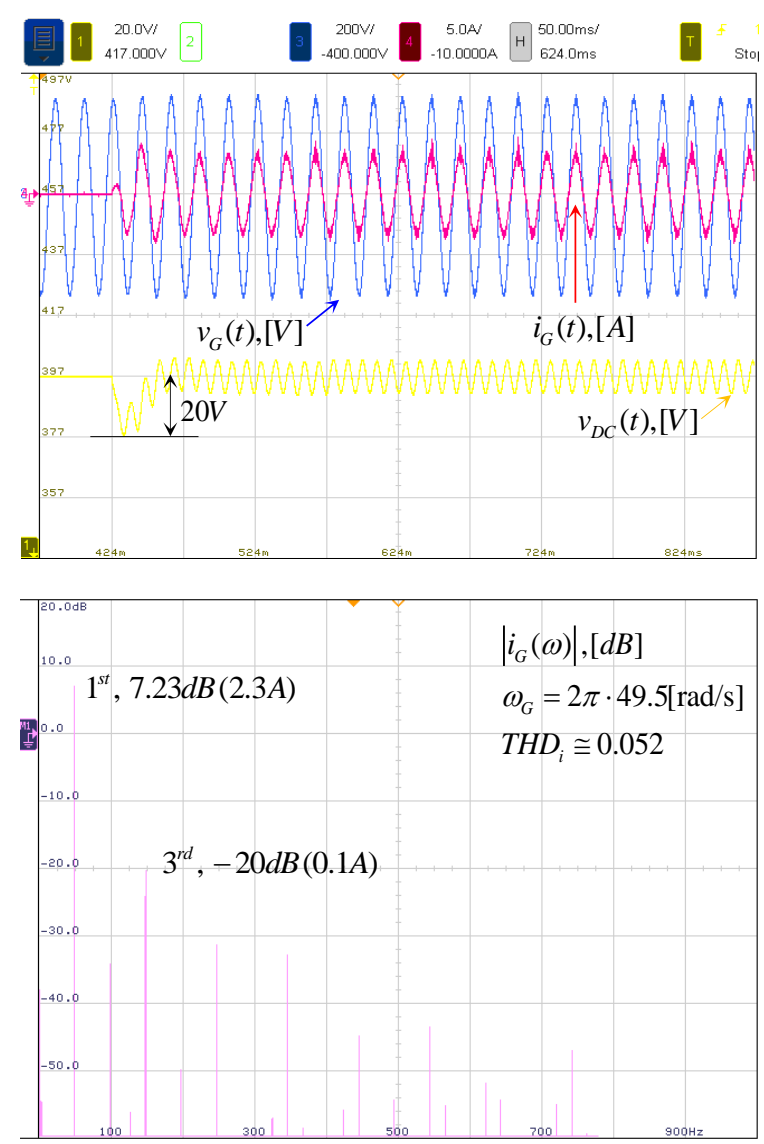

Fig. 6. System response to $0 \%$-to- $100 \%$ load step with PI+Notch controller.

\section{REFERENCES}

[1] S.-H. Jo, S. E. Son and J.-W. Park, "On improving distortion power quality index in distributed power grids," IEEE Trans. Smart Grid, vol. 4, no. 1 , pp. 586 - 595, Mar. 2013.

[2] M. A. Vitorino et al., "Low-frequency power decoupling in single-phase applications" A comprehensive overview," IEEE Trans. Power Electron., vol. 32, no. 104, pp. 2892 - 2912, Apr. 2017.

[3] A. Mutovkin et al., "Control of direct voltage regulated active DC link capacitance reduction circuits to allow plug-and-play operation," IEEE Trans. Ind. Electron., vol. 66, no. 8, pp. 6527-6537, Aug. 2019.

[4] S. Gulur, V. M. Iyer and S. Bhattacharaya, "A dual-loop control structure with improved disturbance rejection for grid-connected converters," IEEE Trans. Power Electron., vol. 34, no. 10, pp. 10233 - 10244, Oct. 2019.

[5] A. Mallik, J. Lu, and A. Khaligh, "A comparative study between PI and type-II compensators for H-bridge PFC converter," IEEE Trans. Ind. Appl., vol. 54, no. 2, pp. 1128-1135, Mar. 2018.

[6] J. Sebastian et al., "On the maximum bandwidth attainable by power factor correctors with a standard compensator," IEEE Trans. Ind. Appl., vol. 46, no. 4, pp. 1485- 1497, Aug. 2010.

[7] S. F. Zarei et al., "DC-link loop bandwidth selection strategy for gridconnected inverters considering power quality requirements," Int. J. Electr. Power Energy Syst., vol. 119, 105879, 2020.

[8] M. Karimi-Ghartemani, S. A. Khajehoddin, P. K. Jain and A. Bakhshari, "A systematic approach to DC-bus control design in single-phase gridconnected renewable converters," IEEE Trans. Power Electron., vol. 28 , no. 7, pp. 3158 - 3166, Jul. 2013.

[9] P. Strajnikov and A. Kuperman, "Guidelines for voltage controller coefficients design to attain prescribed grid current THD and DC link voltage undershoot values in power factor correction front ends," IEEE J. Emerg. Sel. Top. Power Electron., DOI: 10.1109/JESTPE.2021.3100484.

[10] A. El Aroudi, A. Cid-Pastor and L. Martinez-Salamero, "Suppression of line frequency instabilities in PFC AC-DC power supplies by feedback notch filtering the pre-regulator output voltage," IEEE Trans. Circ. Syst. - I: Reg. Papers, vol. 60, no. 3, pp. 796 - 809, Mar. 2013.

[11] R. W. Erickson and D. Maksimovic, Fundamentals of Power Electronics, Kluwer Academic, 2001. 